\title{
Infants' observing and heart period responses as related to novelty of visual stimuli'
}

\author{
WILLIAM J. MEYERS AND GORDON N. CANTOR \\ INSTITUTE OF CHILD BEHAI'IOR AND DEVELOPMENT, UNIVERSITY OF IOWA
}

No justification was found for computing an average reliability estimate although fairly high interobserver correlations were obtained for judgments of infants' fixation times. No intrastimulus decrement was found in observing times, whereas heart period responses when adjusted for prestimulus levels showed across-trial changes, but differentially for males and females. Relationships between observation duration and degree of interstimulus novelty were contingent upon stimulus sequence and tended to be nonmonotonic.

In this initial experiment with infants on the psychophysiology of visual stimulus novelty, both observing and cardiovascular responses were recorded to determine if these were systematically related to novelty, one of Berlyne's (1960) "collative" properties. Two conceptualizations of novelty are used here. The first, "intrastimulus novelty," involves repeated presentation of a given stimulus; degree of novelty is assumed to decrease with repeated exposure of this stimulus. The effects of such a procedure, if they occur, are often termed habituation, or more descriptively, response decrement. The second notion, "interstimulus novelty," entails a comparison of different stimuli, some having been familiarized to various degrees. The present study had two major purposes: (a) to assess interobserver reliability in judging infants' ocular fixation responses; and (b) to examine the relationship of intrastimulus and interstimulus novelty to observing time scores and to cardiac reactivity.

Method

The results are based on data obtained from $24 \mathrm{Ss}$, 12 males and 12 females, each 5 months old ( \pm 1 week).

$S$ sat in an infant seat facing a 12-1/2 x 12-1/2 in. projection screen located $24 \mathrm{in}$, away. The stimulicolored pictures of a ball, a bear, a clown, and a dollwere projected onto the screen by a Dunning Animatic strip film projector. The objects occupied approximately $50 \mathrm{sq}$. in. of the $120 \mathrm{sq}$. in. light field illuminated by the projector. Two peep holes in the screen, 6 in. apart, allowed two observers to view the infant.

The EKG was transduced from chest and back leads into a Fels Cardiotachometer to monitor heart rate. A pulse coincident with the $\mathrm{R}$-wave triggered a Hewlett-Packard counter and printer which timed the successive $\mathbf{R}-\mathbf{R}$ intervals or heart periods and printed them with stimulus codes.

Interstimulus novelty was varied by presenting the clown, ball, bear, and doll pictures differential numbers of times. A given stimulus was projected for four trials (phase 1). Then a new stimulus was introduced and the two stimuli were each presented four times (phase 2). Subsequently, a third stimulus was added (phase 3), and finally a fourth (phase 4), each stimulus appearing four times within a given phase. Thus, after 40 trials, the relative presentation frequencies (presumably from the least to the most novel stimulus) were $16,12,8$, and 4 projections. Four counterbalancing groups insured that each of the four stimuli appeared for the first time in each of the four phases. A 9 sec. stimulus-on and $8 \mathrm{sec}$. stimulus-off schedule was used throughout. One of three pairs of judges observed one-third of the Ss within each of the four counterbalancing groups. Each observer independently depressed a silent switch whenever the infant appeared to be fixating the screen, thus recording total observation time in $.01 \mathrm{sec}$.

\section{Results and Discussion}

Observer reliability. Correlations computed between the 40 pairs of fixation time scores generated by the two observers for each $S$ ranged from .65 to .99 , with a median of .93 . Sixteen of the $24 \mathrm{r}$ 's were .90 and above, six were between .80 and .89 , and two were less than .80 . A technique provided in Snedecor (1956) tests the hypothesis that several r's are from a common population; failure to reject such a hypothesis indicates the legitimacy of computing an average $r$ based on the individual $r$ values. This hypothesis was found untenable $(p<.05)$ for the Ss as a whole, for the two sex groups separately, and for the three groups observed by the different pairs of judges. Consequently, there was no justifiable basis for computing average $r$ values based upon any of these categories. Thus, although the median interobserver reliability was as high as those reported in most studies, the results indicate that a reliability problem might exist in comparable situations. If a criterion of .90 is set as adequate, judgments on only 16 of 24 Ss attained an acceptable level of reliability. More important, statistical demonstration of the legitimacy of averaging $r$ values across Ss was not obtained. This points to the need for explicitly indicating how reliability indices are determined and whether an average $r$ may justifiably be used to represent the overall level of reliability.

Observing time. Two analyses of variance, Lindquist (1953) Type III, were run on the observing time scores (averaged for both observers and blocked over four trials) with sex and counterbalancing groups as betweenSs factors in both analyses. The within-Ss factor in the intrastimulus analysis was phases; in this case, only those scores pertaining to whichever stimulus appeared in all four phases for a given $\mathrm{S}$ were utilized. 


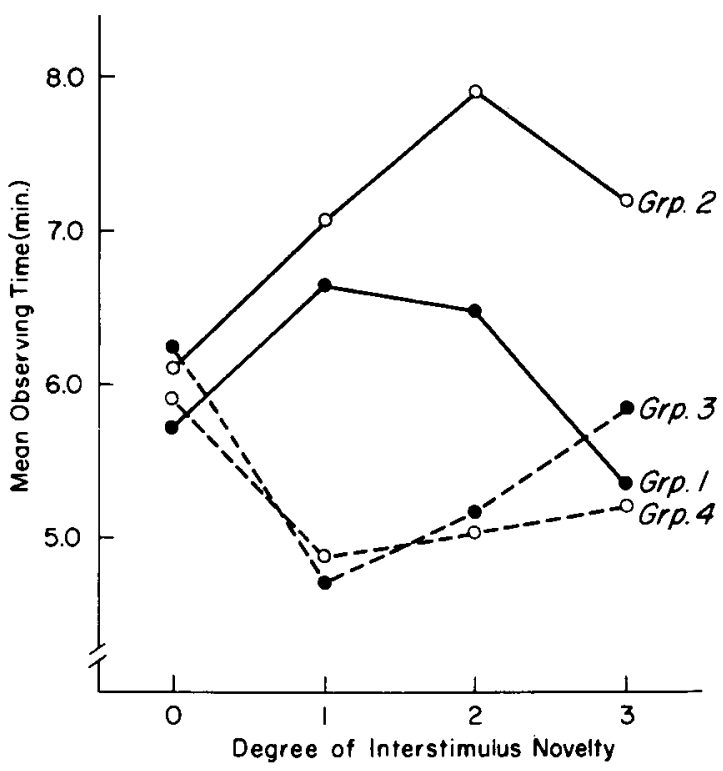

Fig. 1. Observing times for each counterbalancing group as a function of interstimulus novelty. The stimuli corresponding to the $0,1,2$, and 3 degrees of novelty were: Grp. 1-C, D, Br, B; Grp. 2-B, C, D, Br; Grp. 3-Br, B, C, D; Grp. 4-D, Br, B, C. C = clown, $\mathbf{D}=$ doll, $\mathrm{Br}=$ bear, $\mathbf{B}=$ ball .

The within-Ss factor in the interstimulus novelty analysis was degree of interstimulus novelty; here, only those scores obtained in phase 4 for each of the four stimuli were utilized.

In the intrastimulus analysis, no significant $F$-ratios were found for the main effects of sex, counterbalancing groups, phases, or the interactions between them. Thus, decrements in fixation time failed to occur. Our failure to obtain a familiarization effect contrasts with recent findings of observation time decrements (Fantz, 1964; Saayman, Ames, \& Moffett, 1964).

In the interstimulus novelty analysis, only the interaction between degree of novelty and counterbalancing groups was significant $(F=2.68, d f=9 / 48, p<.05)$. This result is illustrated in Fig. 1. Nonmonotonic relations are suggested between observation duration and degree of novelty for each of the groups. Note also that this relation takes opposite directions of curvature for groups 2 and 3 vs. groups 1 and 4 .

Heart period. The heart period data were analyzed as above, using change scores based upon the mean of the three longest during-stimulus $R-R$ intervals (after $\mathrm{S}$ had fixated the stimulus) minus the mean of the three prestimulus $R-R$ intervals. There were no significant intrastimulus or interstimulus novelty effects using raw change scores. If Wilder's (1958) "law of initial value" (LIV) held here, novelty effects may have been masked by prestimulus variability. Correlations were computed between prestimulus levels and change scores for each trial across Ss. These were averaged over trials, after the Snedecor test indicated such averaging to be justified, and modest support was revealed for the LIV (avg. $r=-.18, t=4.86, d f=38, p<.001$ ). In view of this, the heart period analyses were repeated using autonomic lability scores (Lacey, 1956) based upon $r_{x y}$ values computed for each trial across Ss. The interstimulus analysis yielded no significant effects. A significant phases by sex interaction $(F=2.94, \mathrm{~d} f=3 / 18$, $\mathrm{p}<.05$ ) was found in the intrastimulus analysis. The two sexes exhibited approximately equivalent scores during the initial two phases, i.e., increased heartperiods or heart rate decelerations accompanying stimulus presentation. However, during the last two phases, opposite tendencies were seen for the two sexes; males showed response increments (increased heart rate decelerations), whereas females showed response decrements (decreased heart rate decelerations). These heart period results suggest that cardiac changes may be sensitive to intrastimulus novelty manipulations but that control of prestimulus levels maybe necessary to demonstrate such effects. While heart rate decelerations apparently accompany the observation of visual stimuli by infants, the potential relevance of sex differences cannot be overlooked, especially with repeated stimulus presentation. Finally, it appears that observing time and autonomic responses may be differentially sensitive to the two types of novelty manipulations distinguished here.

\section{References}

Berlyne, D. E. Conflict, arousal, and curiosity. New York: McGrawHill, 1960.

Fantz, R. L. Visual experience in infants: decreased attention to familiar patterns relative to novel ones. Science, 1964, 146, 668-670.

Lacey, J. I. The evaluation of autonomic responses: toward a general solution. Ann. New York Acad. Sci., 1956, 67, 123-164.

Lindquist, E. F. Design and analy sis of experiments in psychology and education. Boston: Houghton Mifflin Co., 1953.

Saayman, G., Ames, Elinor W., \& Moffett, Adrienne. Response to novelty as an indicator of visual discrimination in the human infant. J. exp. child Psychol., 1964, 1, 189-198.

Snedecor. G. W. Statistical methods, 5th edition. Ames, Iowa: The Iowa State College Press, 1956. Pp. 178-180.

Wilder, J. Modern psychophysiology and the law of initial value. Amer. J. Psychotherapy, 1958, 12, 199-221.

\section{Note}

1. Supported by Public Health Service research grant HD-00892, National Institute of Child Health and Human Development, formerly MH-06624, National Institute of Mental Health. Observers were Gary Gullickson, David R. Pederson, and Miss Leslie Segner. The interobserver reliability results were previously presented at the 1965 meeting of the Society for Research in Child Development at Minneapolis. 